\title{
ON THE FACTORS AND FIBERINGS OF MANIFOLDS
}

\author{
GAIL S. YOUNG, JR.
}

1. Introduction. In this note I propose to discuss first some of the properties of factors of topological manifolds, possibly having boundaries. Particular emphasis is placed on the 1- and 2-dimensional factors, which are shown to be manifolds themselves, thus generalizing slightly results of Borsuk [2]. ${ }^{1}$ These results are then applied to fiber bundles, and to the question as to whether $E^{n}$, Euclidean $n$-space, is a fiber bundle with a compact fiber. As a preliminary, I prove an elementary theorem which asserts that if a fiber bundle is connected and locally connected, then the components of the fiber are all homeomorphic, and which apparently has escaped notice. From this, the other theorems of this paper, and known results, follow easily several results concerning impossible fiberings of $E^{n}$. In particular it is shown that, except for possibly a finite set as fiber, there is no compact fibering of $E^{z}$ or $E^{4}$, or of $E^{5}$, one possibility excepted.

Many of the proofs are modifications of arguments due to Borsuk [2] and Montgomery and Samelson [7]. Several conversations with Samelson were helpful and stimulating.

2. 1- and 2-dimensional factors of manifolds. The first two theorems of this section are extensions of Theorems 3 and 13 of Borsuk's [2] and lean heavily on his arguments. The ingenious use of the Brouwer theorem on invariance of domain in Theorem 1, originating with Borsuk, was also discovered practically simultaneously by Szumbarski [10], and used for a similar purpose.

THEOREM 1. If $A \times B$ is an n-manifold ${ }^{2}$ (possibly with boundary) and some 0-dimensional subset of $A$ separates an open subset of $A$, then $A$ is homeomorphic to a connected subset of a simple closed curve.

Proof. We recall that since $A \times B$ is locally connected, so are $A$ and

Presented to the Society, April 17, 1948, under the title On product manifolds and fiberings; received by the editors November 20, 1948.

${ }_{1}$ Numbers in brackets refer to the bibliography at the end of the paper.

${ }^{2}$ A connected space $M$ is an $n$-manifold if every point of $M$ has a neighborhood which is homeomorphic to Euclidean $n$-space $E^{n}$, or to the set of all points in $E^{n}$ whose $n$th coordinate is non-negative. The set of all points in $M$ not lying in neighborhoods of the first kind constitute the boundary of $M$. We use $A \times B$ for the topological product of two spaces. 
$B$. Let $D$ be a connected open subset of $A$, and suppose that the 0 -dimensional subset $T$ of $D$ separates it. By a consequence of local connectivity [12; III, 1.4], we can assume that $T$ is closed in $D$, so that $T \times B$ is closed in $D \times B$ and separates it. Since no subset of an $n$-manifold of dimension less than $n-1$ separates any open subset of it, $\operatorname{dim} T \times B \geqq n-1$. No point of $T$ is an interior point, so that neither is any point of $T \times B$. Hence $\operatorname{dim} T \times B \neq n$ [5; Theorem IV, 3$]$, and must be $n-1$. But, by [5; Theorem III, 4], $\operatorname{dim} B \leqq \operatorname{dim} T \times B$ $\leqq \operatorname{dim} T+\operatorname{dim} B=\operatorname{dim} B$, so that $\operatorname{dim} B=n-1$. If $A$ is not homeomorphic to a subset of a circle, $A$ contains a simple triod $a x \cup b x \cup c x$. Since $a x$ is 1-dimensional, $\operatorname{dim} a x \times B=n$, by [6], and hence there is a point $(u, v)$ in $a x \times B$ interior to this set, but not lying in the boundary $\beta(A \times B)$ of the manifold $A \times B$. There is a homeomorphism $h$ : $a x \rightarrow a x b$, sending $u$ onto $x$, which induces a homeomorphism $h^{\prime}: a x \times B \rightarrow a x b \times B$, defined by $h^{\prime}(p, q)=(h(p), q)$. By the Brouwer theorem on invariance of domain, $h^{\prime}(u, v)=(x, v)$ is in (Int $a x b \times B$ ) $\cap[A \times B-\beta(A \times B)]$. But $(x, v)$ is a limit point of $(c x-x) \times B$, giving a contradiction.

Theorem 2. Let $A \times B$ be an n-manifold, with $\operatorname{dim} A=1,2$. Then $A$ is a manifold, possibly with boundary, though having a boundary only if $A \times B$ does.

Proof. If $\operatorname{dim} A=1$, that $A$ is a manifold follows from Theorem 1. To show this if $\operatorname{dim} A=2$, we need only show that $A$ has no local cut point, and that given a point $a$ in $A$, there is an open set $V$ containing $a$ such that every simple closed curve in $V$ separates $A$, by [16; Theorem 1.1]. The first condition follows from Theorem 1, and the second condition can be obtained from Borsuk's argument for his Theorem 13, where he proves the same thing for a manifold without boundary. Only minor modifications are needed to take care of the complications introduced by the boundary.

If $A$ has a boundary, $\beta(A)$, then $B \times \beta(A)$ is an (n-1)-dimensional closed subset of $A \times B$ separating no open set, which is impossible if $\beta(A \times B)$ is empty. This last remark also proves that no manifold with boundary is a factor of a manifold without boundary, which must be well known.

M. Szumbarski has proved in [10] that every 1-dimensional factor of a closed $n$-cell, $I^{n}$, is a 1-cell, and that every factor of $I^{n}$, for $1 \leqq n \leqq 3$, is itself a cell. In the second of these statements, if $I^{n}$ $=A \times B$, and $n \leqq 3$, then $\operatorname{dim} A, \operatorname{dim} B \leqq 2$ (neglecting the trivial case of $A$ or $B$ being a point). Hence both of these propositions are implied by the following corollary of Theorem 2 . 
COROLlaRY 2.1. Every 1- or 2-dimensional factor of a closed n-cell is itself a closed cell.

Proof. If $I^{n}=A \times B$, each factor is homologically trivial, by the Künneth formula. Hence if $\operatorname{dim} A=1, A$ is a closed 1-cell, since it cannot be a 1 -sphere. If $\operatorname{dim} A=2, A$ must be a 2 -manifold with boundary, since no compact 2-manifold without boundary is homologically trivial. But the only homologically trivial 2-manifold with boundary is the closed 2-cell.

Quite possibly every factor of a closed $n$-cell is itself a cell. However, J. H. C. Whitehead has found [11] two non-homeomorphic 2-manifolds with boundary, such that the product spaces obtained by multiplying each with an interval are homeomorphic, so that such a conjecture does not carry overwhelming conviction.

3. Factors with retraction properties. In this section I show that no manifold without boundary has as factor any set of a rather improbable type.

THEOREM 3. If $A \times B$ is an open subset of a closed orientable n-manifold $M$ (without boundary), and $A$ is compact, then no nowhere dense subset of $A$ is a deformation retract of $A$.

Proof. Suppose that the theorem is false. Then $A$ contains a nowhere dense set $R$ which is a deformation retract of $A$. If $U$ is a small open subset of $B$ with compact closure, then $A \times \beta(U)$ not only separates $A \times B$, but also separates $M$, as $A \times \bar{U}$ is compact. Now Wilder has proved [12, Theorem VII, 3.9] that if, in $M$, the $(n-1)$ st Betti number of a closed subset $T, p_{n-1}(T)$, is $k$, then $M-T$ has at most $k+1$ components. Hence $p_{n-1}[A \times \beta(U)] \geqq 1$. I shall show that at least for some set $U$ as described, $p_{n-1}[A \times \beta(U)]=0$, thus getting a contradiction.

There is a deformation retraction $r: A \times I \rightarrow R$ satisfying $r(a, 0)$ $=a, r(x, t)=x, x$ in $R$, and $r(A, 1)=R$. For any set $U$, described above, this induces a deformation retraction $r^{\prime}:[A \times \beta(U)] \times I \rightarrow R$ $\times \beta(U)$ by the equation $r^{\prime}[(a, b), t]=[(r(a), b), t], a$ in $A, b$ in $\beta(U)$, $t$ in $I$. Then $r^{\prime}$ induces a homomorphism $r^{\prime}: H_{q}[A \times \beta(U)]$ $\rightarrow H_{q}[R \times \beta(U)]$, these being homology groups, which is well known to be an isomorphism onto. Hence if $\operatorname{dim} R \times \beta(U)<n-1$, implying $p_{n-1}[R \times \beta(U)]=0$, the proof would be complete. However, this fact is not obvious, and an analogous statement is not true for product spaces in general. Suppose, then, that for every $U$ in $B$ satisfying our conditions, $\operatorname{dim} R \times \beta(U)=n-1$. Then there exists an uncountable collection, $G$, of such neighborhoods, the boundaries of no two inter- 
secting. ${ }^{3}$ Each set $R \times \beta(U)$ contains a Cantorian $(n-1)$-dimensional manifold, by [5; VI 8$]$, and which, by [1], therefore separates some open $n$-cell in $A \times B$. It is not hard to see that some open $n$-cell $D$ in $A \times B$ is separated by uncountably many sets $[R \times \beta(U)] \cap D$. By Whyburn's [12; III, 2.2], there is an uncountable collection $H$ of such sets forming a saturated collection; that is, given any point $x$ in $D$, and a set $X$ in $H$ not containing $x$, some other set in $H$ separates $x$ from $X$ in $D$. However, for each set $[R \times \beta(U)] \cap D$ in $H$, there is a point $a$ in $R$ which is arcwise accessible from $A-R$ by an arc $T$, and which is such that, for some $b$ in $\beta(U), T \times b$ is in $[A \times \beta(U)] \cap D$. It follows that each point of $T \times b-a \times b^{\prime}$ is separated from $[R \times \beta(U)]$ $\cap D$ in $D$ by some element of $H$. But each two sets $A \times \beta(U)$, $A \times \beta\left(U^{\prime}\right), U$ and $U^{\prime}$ in $G$, are mutually exclusive, which gives a contradiction.

Theorem 3 says, roughly speaking, that a set which is too much like a manifold with boundary cannot be a factor of a manifold without boundary, thus generalizing the statement following Theorem 2 . Some light may be thrown on the proof by the following example. Pontrjagin [8] constructs two compact 2-dimensional spaces, $A$ and $B$, such that $\operatorname{dim} A \times B=3$. Add a countable sequence of arcs, $\left\{T_{i}\right\}$, to $A$, each having only an end point in common with $A$, with $U T_{i}$ dense in $A$, and with $\lim \operatorname{diam} T_{i}=0$. Let $A^{\prime}=A \cup \cup T_{i}$. Then $A$ is a deformation retract of $A^{\prime}$, and is nowhere dense in $A^{\prime}$. But dim $A^{\prime} \times B=\operatorname{dim} A \times B$.

4. Fiber bundles. Primarily to fix the notation, I shall state the definition of fiber bundle. A fiber bundle is the totality consisting of (a) three spaces $A, B$, and $F$, called the bundle, the base space, and the fiber, respectively; (b) a topological group $G$ of homeomorphisms of $F$ onto itself, called the structure group; (c) a continuous map, $p: A \rightarrow B$, called the projection, with the property that for each $b$ in $B, p^{-1}(b)$ is homeomorphic to $F$; (d) a covering $\left\{U_{\alpha}\right\}$ of $B$ by open sets called admissible neighborhoods, and a family of homeomorphisms $\phi_{\alpha}: U_{\alpha}$ $\times F \rightarrow p^{-1}\left(U_{\alpha}\right)$ such that $p \phi_{\alpha}(b, f)=b$, for each $(b, f)$ in $U_{\alpha} \times F$. These entities must also satisfy the following condition: For $b$ fixed and each $\alpha$ such that $U_{\alpha}$ contains $b$, consider the map $\phi_{\alpha b}: F \rightarrow p^{-1}(b)$ defined by $\phi_{\alpha b}(f)=\phi_{\alpha}(b, f)$. If $b$ is in $U_{\alpha} \cap U_{\beta}$, let $g_{\alpha \beta}=\phi_{\beta b}^{-1} \phi_{\alpha b}$. Then $g_{\alpha \beta}$ is a homeomorphism of $F$ onto $F$, and we require this to be in $G$. It was pointed out in [3] that for $G$ the group of all homeomorphisms of $F$ onto $F$, the definition can be simplified to (a), (c), and (d). The above notation will be used without explicit mention. [14].

${ }^{3}$ From this point on, the argument is a generalization of that given in my paper 
5. Conditions for components of a fiber to be homeomorphic. I prove several simple results, that have apparently escaped notice, concerning fibers.

THEOREM 4. If the fiber bundle $A$ is a connected topological space, and the fiber $F$ is the union of two separated sets, $F_{1}$ and $F_{2}$, then some element of $G$ maps a point of $F_{1}$ into $F_{2}$.

Proof. Suppose the theorem is false. Let $D_{i}, i=1,2$, be the set of all points $a$ in $A$ such that for some $U_{\alpha}$ containing $p(a)=b, \phi_{\alpha b}^{-1}(a)$ is in $F_{\boldsymbol{i}}$. This definition is independent of the particular choice of $U_{\alpha}$. For, if $U_{\beta}$ contains $b$, since $\phi_{\alpha b}\left(F_{i}\right)$ contains $a$, and since $\phi_{\beta b}^{-1} \phi_{\alpha b}\left(F_{i}\right)$ is in $F_{i}$ (if for some $g$ in $G, g\left(F_{2}\right)$ meets $F_{1}$, then $g^{-1}\left(F_{1}\right)$ meets $F_{2}$ ), we have $\phi_{\beta 0}^{-1}(a)$ is in $F_{i}$. Clearly, $D_{1} \cap D_{2}=0$. Now $U_{\alpha} \times F_{i}, i=1,2$, is open in $U_{\alpha} \times F$, which is homeomorphic to $p^{-1}\left(U_{\alpha}\right)$, so $D_{i} \cap p^{-1}\left(U_{\alpha}\right)$ is open in $A$. But $D_{i}=\cup_{\alpha} D_{i} \cap p^{-1}\left(U_{\alpha}\right)$, so that $D_{i}$ is open in $A$. This implies that $D_{1}$ and $D_{2}$ form a separation of $A$, whereas $A$ is connected.

Theorem 5. If the fiber bundle $A$ is a connected topological space, and each component of $F$ is open in $F$, then each two components of $F$ are homeomorphic, and the structure group $G$ acts transitively on the components of $F$.

Proof. Let $F_{1}$ be the union of a component of $F$ and all components of $F$ homeomorphic to it, and let $F_{2}=F-F_{1}$. Then $F_{1}$ and $F_{2}$ are mutually separated. By Theorem 4, some element $g$ of $G$ maps a point of $F_{1}$ into $F_{2}$. But then some component of $F_{1}$ is mapped homeomorphically onto a component of $F_{2}$ by $g$. This is impossible, so that $F_{2}=0$.

If the structure group $G$ does not act transitively on the components of $F$, then $F$ is the union of two separated sets $F_{1}$ and $F_{2}$ where no element of $G$ maps a component of $F_{1}$ onto a component of $F_{2}$. This again contradicts Theorem 4 .

CoRollary 5.1. If the fiber $F$ has only a finite number of components, or if $A$ is locally connected and connected, then the hypothesis on $F$ in Theorem 5 is satisfied, and any two components of $F$ are homeomorphic.

The next result is suggested by the Eilenberg-Whyburn factorization theorem [12; VIII, 4.1]. For economy in space (!), I shall not state the most general hypothesis.

Theorem 6. Let the fiber bundle $A$ be locally compact, metric, and connected. Let the fiber $F$ have only a finite number of components. Let $p=l m$ be the monotone-light factorization of $p$, with $m(A)=C$. Then the map $m$ defines a fibering of $A$ with base space $C$, fiber $F^{\prime}$ a component of 
$F$, projection $m$ and with structure group the group of all homeomorphisms of $F^{\prime}$. The map $l$ defines a fibering of $C$ with base space $B$, fiber a set $l^{-1}(x), x$ in $B$, projection $l$, and with structure group isomorphic to the structure group $G$ of $p$, modulo the subgroup of $G$ consisting of all those elements which leave each component of $F$ fixed; $C$ is a covering space of $B$.

Notice that if we had that $A$ were compact and locally connected, it would follow that $F$ had only a finite number of components.

Proof. By 5.1, all components of $F$ are homeomorphic to one, $F^{\prime}$. The space $C$ is the space of all sets which are components of a set $p^{-1}(x), x$ in $B$, and whose open sets are defined as the set of all such components lying in an open set in $A$. Let $U$ be an admissible neighborhood in $B$. Then $p^{-1}(U)$ is the union of a finite number of open sets, $V_{1}, \cdots, V_{n}$, each homeomorphic to $F^{\prime} \times U$, and each teing unions of components of sets $p^{-1}(x)$. Considering these components as points of $C$, the map $l: C \rightarrow B$ is $1-1$ and continuous on each set $V_{i}^{\prime}$, where $V_{i}^{\prime}$ is the open set in $C$ whose elements are subsets of $V_{i}$. It is easy to see that $l$ is a homeomorphism on each $V_{i}^{\prime}$, so that $C$ is a covering space of $B$, and $l$ is a fibering of $C$. The statement concerning the structure group for $l$ follows from Theorem 5 .

Using the information about the sets $V_{i}$ obtained from $p$, it is easy to see that $m: A \rightarrow C$ is a fibering as described, using the remark at the end of $\$ 4$.

6. Compact fiberings of $E^{n}$. We are now ready to apply the above results to the question as to whether $E^{n}$, Euclidean $n$-space, can be a fiber bundle with a compact fiber, raised by Montgomery and Samelson in [7].

THEOREM 7. There is no compact fibering of $E^{n}$ by a fiber, one component of which is a $k$-sphere, or a $k$-torus, or a set of the type described in Theorem 3.

Proof. This is a consequence of Theorems 5, 6 and the fact that (a) as Montgomery and Samelson point out, Gysin's methods [4] show that $E^{n}$ cannot be fibered with a sphere as fiber; (b) Eckmann, Samelson, and Whitehead [3] have proved that there is no such fibering with a $k$-torus as fiber; and (c) Theorem 3.

THEOREM 8. There is no fibering of $E^{n}, n>1$, by a compact fiber of dimension 1 or $n-1$.

Proof. We may assume, from Theorem 6 , that $F$ is connected. For 
$\operatorname{dim} F=1$, the theorem is a consequence of Borsuk's result referred to above (and generalized in Theorem 2), and Theorem 7 .

If $\operatorname{dim} F=n-1$, then $\operatorname{dim} B=1$. For if $\operatorname{dim} B>1, B$ contains uncountably many mutually exclusive compact sets $\left\{C_{\alpha}\right\}$ with $\operatorname{dim}$ $C_{\alpha}=1$, by $[5 ;$ VII, 5$]$. Then by Hurewicz's theorem, $\operatorname{dim} C_{\alpha} \times F=n$, so that each set $C_{\alpha} \times F$ has interior points. But this contradicts the separability of $E^{n}$. Hence $B$ is either a simple closed curve or an open curve, since by Borsuk's result, it is locally Euclidean. Now if $x$ is in $B$, then $p^{-1}(x)$ separates some open subset of $E^{n}$ containing it, and hence separates $E^{n}$ into two sets each of which is an inverse set under $p^{-1}$. It follows that $B$ is not a simple closed curve. On the other hand, since $p^{-1}(x)$ is compact, one of its two complementary domains has a compact closure. Hence so does one component of $B-x$. But then $B$ cannot be an open curve.

COROLLARY 8.1. There is no fibering of $E^{2}$ or $E^{3}$ by a compact fiber of dimension greater than 0.

In [15] I gave a general theorem on interior mappings of $E^{2}$ from which the part of 8.1 concerning $E^{2}$ followed. As shown there, for $E^{2}$ and the case where the dimension of the fiber is 0 , results of Whyburn prove that this is also impossible. For $E^{n}, n>2$, the case of a compact 0-dimensional fiber is quite difficult and includes the following: If $f: E^{n} \rightarrow E^{n}$ is periodic with period $k$, then not every orbit of a point under $f$ has exactly $k$ points. (If for some such mapping, every orbit had $k$ points, it is easy to see that $E^{n}$ is a covering space of the orbit space, so that it is a fiber bundle with an orbit as fiber.)

CoROLlary 8.2. There is no fibering of $S^{n}, n>1$, by a fiber of dimension $n-1$.

Proof. Except that $B$ cannot conceivably be an open curve, the proof is just like Theorem 8 .

THEOREM 9. There is no compact fibering of $E^{n}, n>2$, by a fiber $F$ of dimension $n-2$ onto a base space $B$ of dimension 2 .

Proof. Suppose again that $F$ is connected. Since $\operatorname{dim} B=2$, it follows from Borsuk's theorem that $B$ is a 2-manifold. I now show that $B$ is a plane, by showing that it is simply connected, the plane being the only non-compact simply connected 2 -manifold without boundary. Let $f: I \rightarrow B$ be a closed path, $I$ being the unit interval. Without loss, we can assume that $f$ is constant over $[1 / 2,1]$. By covering homotopy [9], and arcwise connectivity of $F$, there is a closed path 
$\phi: I \rightarrow E^{n}$ such that $p \phi=f$. Since $E^{n}$ is simply connected, $\phi$ is homotopic to a constant mapping, so that so is $f$ by the homotopy induced in $B$ by $p$.

Let $U$ be an admissible 2-cell neighborhood in $B$, and let $J$ be a simple closed curve in $U$. Then $J$ separates $B$ into two connected sets, so that $p^{-1}(J)$ separates $E^{n}$ into two connected sets. By the Alexander duality theorem, $b_{n-1}\left[p^{-1}(J)\right]=1, b_{k}$ denoting the $k$ th Betti number. Since $U$ is admissible, $p^{-1}(J)$ is homeomorphic to $J \times F$, and by the Künneth formula we must have $b_{n-2}(F)=1$. It follows that for any point $x$ in $B, b_{n-2}\left[p^{-1}(x)\right]=1$, so that in $E^{n}$ $-p^{-1}(x)$ there is a (singular) 1 -cycle, $Z^{1}$, linked with $p^{-1}(x)$. Add a point $\omega_{n}, \omega_{2}$ to each of $E^{n}, B$ to make them both spheres. Consider the singular 1-cycle $p\left(Z^{1}\right)$ in $B$. This lies in $B \cup \omega_{2}-x$, an open 2-cell, so is contractible to $\omega_{2}$ in this set by a homotopy $h(y, t)$. Since covering homotopy holds for compact subsets of $B$, we can move $Z^{1}$ into an arbitrary neighborhood of $\omega_{n}$ by a homotopy covering $h(y, t)$ for some range of $t$. This implies that $Z^{1}$ bounds in $\left(E^{n} \cup \omega_{n}\right)-p^{-1}(x)$, a contradiction.

This argument is quite closely related to Montgomery and Samelson's [7; Lemma 2]. I might note that if $b_{n-2}(F) \neq 0$, it would follow that every sufficiently small simple closed curve in $B$ would separate it, so that $B$ would be a 2 -manifold, and the condition on $\operatorname{dim} B$ could be removed.

Theorem 10. There is no fibering of $E^{n}$ by a fiber $F$ of dimension 2.

Proof. Assuming $F$ connected, it follows from Theorem 2 that it is a 2-manifold. Hence $\operatorname{dim} B=n-2$, and we can assume $\operatorname{dim} B>1$, or the result follows from Theorem 8 . Then $B$ has no local cut points, so that there exists an open set $U$, with $\bar{U}$ in an admissible neighborhood, such that $\beta(U)$ separates $B$ into two connected open sets. Then $b_{n-1}[F \times \beta(U)]=1$, so that $b_{2}(F)=1$. Montgomery and Samelson point out that the fundamental group of $F$ must be abelian, and the only two orientable 2-manifolds without boundary having this property are the sphere and the torus. Hence Theorem 10 follows from Theorem 7 .

CoROllary 10.1. There is no fibering of $E^{4}, E^{5}$ by a compact fiber of dimension greater than 0 , except possibly for $E^{5}$, if $\operatorname{dim} F=3, \operatorname{dim} B>2$.

Proof. Each possible dimension of $F$ is covered by one of Theorems $7,8,9$, and 10 . 


\section{BIBLIOGRAPHY}

1. P. Alexandroff, Analyse géometrique de la dimension des ensembles fermés, C. R. Acad. Sci. Paris vol. 191 (1930) pp. 475-477.

2. K. Borsuk, On the decomposition of manifolds into curves and surfaces, Fund. Math. vol. 33 (1945) pp. 273-298.

3. B. Eckmann, H. Samelson, and G. W. Whitehead, On fibering spheres by toruses, Bull. Amer. Math. Soc. vol. 55 (1949) pp. 433-438.

4. W. Gysin, Zur Homologietheorie der Abbildungen und Faserungen von Mannigfaltigkeiten, Comment. Math. Helv. vol. 14 (1942) pp. 61-122.

5. W. Hurewicz and H. Wallman, Dimension theory, Princeton University Press, 1941.

6. W. Hurewicz, Sur la dimension des produits cartésiens, Ann. of Math. vol. 36 (1935) pp. 194-197.

7. D. Montgomery and H. Samelson, Fiberings with singularities, Duke Math. J. vol. 13 (1946) pp. 51-56.

8. L. Pontrjagin, Sur une hypothèse fondamentale de la theorie de la dimension, C. R. Acad. Sci. Paris vol. 190 (1930) pp. 1105-1107.

9. N. E. Steenrod, The classification of sphere bundles, Ann. of Math. vol. 45 (1944) pp. 294-311.

10. M. Szumbarski, Sur la décomposition des elements euclideans en produits cartesiens, Rec. Math. (Mat. Sbornik) vol. 16 (1945) pp. 39-42.

11. J. H. C. Whitehead, On the homotopy types of manifolds, Ann. of Math. vol. 41 (1940) pp. 823-832.

12. G. T. Whyburn, Analytic topology, Amer. Math. Soc. Colloquium Publications, vol. 28, 1942.

13. R. L. Wilder, Topology of manifolds, Amer. Math. Soc. Colloquium Publications, vol. 32, 1949.

14. G. S. Young, A generalization of $R$. L. Moore's theorem on simple triods, Bull. Amer. Math. Soc. vol. 50 (1944) p. 714.

15. - On compact fiberings of the plane, ibid. vol. 53 (1947) pp. 295-298.

16. - A characterization of 2-manifolds, Duke Math. J. vol. 14 (1947) pp. 979-990.

UNIVERSITY OF MichigAN 